Роман И. Гвоздев, Иван И. Швецов-Шиловский, Сергей Б. Шмаков.

АДАПТИРОВАННАЯ МЕТОДИКА КОНТРОЛЯ ФУНКЦИОНАЛЬНЫХ СБОЕВ

В NOR FLASН-ПАМЯТИ ПРИ ИСПЫТАНИЯХ НА СТОЙКОСТЬ К ВОЗДЕЙСТВИЮ ТЯЖЁЛЫХ ЗАРЯЖЕННЫХ ЧАСТИЦ

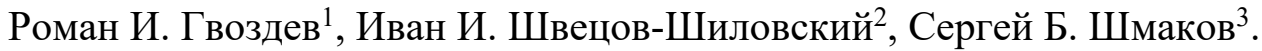 \\ ${ }_{1,2,3}$ Акичонерное общество «Экспериментальное научно-производственное объединение \\ СПЕЦИАЛИЗИРОВАННЫЕ ЭЛЕКТРОННЫЕ СИСТЕМЫ», \\ Каширское ш., 31, Москва, 115409, Россия \\ Ie-mail:rigvoz@spels.ru,http://orcid.org/0000-0002-9284-5785 \\ 2e-mail: iish@spels.ru,http://orcid.org/0000-0002-8161-9926 \\ 3 e-mail:sbshmak@spels.ru,http://orcid.org/0000-0002-4692-9355

\section{АДАПТИРОВАННАЯ МЕТОДИКА КОНТРОЛЯ ФУНКЦИОНАЛЬНЫХ СБОЕВ В NOR FLASН-ПАМЯТИ ПРИ ИСПЫТАНИЯХ НА СТОЙКОСТЬ К ВОЗДЕЙСТВИЮ ТЯЖЁЛЫХ ЗАРЯЖЕННЫХ ЧАСТИЦ DOI: http://dx.doi.org/10.26583/bit.2020.3.06}

Аннотащия. В данной статье предложена адаптированная методика контроля функциональных сбоев в NOR flash-памяти при испытаниях на стойкость к воздействию тяжёлых заряженных частиц. Эксперимент проводился на базе циклотрона У-400. Экспериментальные результаты апробации адаптированной методики показали необходимость разделения функциональных сбоев (ФС) на 5 типов в зависимости от характера каждого ФС и подтверждают необходимость адаптации методики и средств контроля для оценки параметров чувствительности к воздействию тяжёлых заряженных частиц (ТЗЧ) по одиночным радиационным эффектам (ОРЭ) ФС. Полученные результаты предоставляют дополнительные данные о параметрах чувствительности микросхем к воздействию ТЗЧ по ОРЭ производителю аппаратуры для разработки системы парирования наблюдаемых эффектов в аппаратуре, чтобы увеличить безопасность хранящейся информации.

Ключевые слова: функциональный сбой, тяжёлье заряженные частицы, стойкость, микросхема, аппаратно-программный комплекс.

Для иитирования: ГВОЗДЕВ, Роман И.; ШВЕЦОВ-ШИЛОВСКИЙ, Иван И.; ШМАКОВ, Сергей Б. Шмаков. АДАПТИРОВАННАЯ МЕТОДИКА КОНТРОЛЯ ФУНКЦИОНАЛЬНЫХ СБОЕВ В NOR FLASН-ПАМЯТИ ПРИ ИСПЫТАНИЯХ НА СТОЙКОСТЬ К ВОЗДЕЙСТВИЮ ТЯЖЁЛЫХ ЗАРЯЖЕННЫХ ЧАСТИЦ. Безопасность информаиионных технологий, [S.l.], v. 27, n. 3, p. 66-75, 2020. ISSN 2074-7136. Доступно на: <https://bit.mephi.ru/index.php/bit/article/view/1293>. Дата достуna: 02 sep. 2020. DOI: http://dx.doi.org/10.26583/bit.2020.3.06.

Roman I. Gvozdev ${ }^{1}$, Ivan I. Shvetsov-Shilovskiy ${ }^{2}$, Sergey B. Shmakov ${ }^{3}$

1,2,3 Joint Stock Company "Experimental Research and Production Association SPECIAL ELECTRONIC SYSTEM",

Kashirskoe sh., 31, Moscow, 115409, Russia

Ie-mail:rigvoz@spels.ru,http://orcid.org/0000-0002-9284-5785

${ }^{2}$ e-mail: iish@spels.ru,http://orcid.org/0000-0002-8161-9926

3e-mail: sbshmak@spels.ru,http://orcid.org/0000-0002-4692-9355

\title{
Adapted method for monitoring functional failures in NOR FLASH-memory during tests for resistance to heavy charged particles DOI: http://dx.doi.org/10.26583/bit.2020.3.06
}

Abstract. This study offers an adapted method for monitoring functional failures in NOR flash memory during tests for resistance to heavy charged particles. The experiment was conducted on the basis of the "U-400" cyclotron. Experimental results of approbation of the adapted method have shown the need to divide functional failures (FF) into 5 types depending on the nature of each FF and confirm the need to adapt the methodology and monitoring tools to assess the parameters of sensitivity to the effects of heavy charged particles (HCP) on single radiation effects (SRE) of the FF. The obtained results provide additional data on the parameters of the sensitivity of chips to the effects of HCP on the SRE to the 
Роман И. Гвоздев, Иван И. Швецов-Шиловский, Сергей Б. Шмаков.

АДАПТИРОВАННАЯ МЕТОДИКА КОНТРОЛЯ ФУНКЦИОНАЛЬНЫХ СБОЕВ

В NOR FLASН-ПАМЯТИ ПРИ ИСПЫТАНИЯХ НА СТОЙКОСТЬ

К ВОЗДЕЙСТВИЮ ТЯЖЁЛЫХ ЗАРЯЖЕННЫХ ЧАСТИЦ

equipment manufacturer for the development of a system for parrying the observed effects in the equipment in order to increase the security of stored information.

Keywords: functional failures, heavy charged particles, resistance, chip, a hardware-software complex.

For citation: GVOZDEV, Roman I.; SHVETSOV-SHILOVSKIY, Ivan I.; SHMAKOV, Sergey B. Adapted method for monitoring functional failures in NOR FLASH-memory during tests for resistance to heavy charged particles. IT Security (Russia), [S.l.], v. 27, n. 3, p. 66-75, 2020. ISSN 2074-7136. Available at: <https://bit.mephi.ru/index.php/bit/article/view/1293>. Date accessed: $02 \quad$ sep. 2020. DOI: http://dx.doi.org/10.26583/bit.2020.3.06.

\section{Введение}

Особое значение среди факторов, влияющих на космические аппараты, имеет воздействие полей ионизирующих излучений (ИИ) космического пространства электронов, протонов, ионов [1]. ИИ создаётся ядерными частицами, к основным из них относятся: ионы, нейтроны, протоны, гамма-кванты электроны и фотоны. Ядерные частицы можно разделить на незаряженные (нейтроны, гамма-кванты, фотоны) и заряженные (протоны, ионы, электроны). К ТЗЧ относятся протоны и ионы [2].

Одиночные ядерные частицы (ОЯЧ) вызывают эффекты в интегральных схемах изза локального энерговыделения в микрообъёме элемента интегральной схемы [2]. Возникающие эффекты приводят к нарушению работоспособности и изменению значений параметров электронной компонентной базы, комплектующих космической аппаратуры, для которых проблема ОРЭ стоит наиболее остро [3-4]. Выделившаяся энергия преобразуется в неравновесный заряд, который собирает чувствительная область. Заряд из чувствительной области преобразуется в «помехи» (токи, напряжения), из-за которых развивается ОРЭ $[2,5,6]$.

В настоящее время наиболее критичным радиационным эффектом в интегральных схемах электронной аппаратуры космического назначения из-за воздействия отдельных ТЗЧ стали ОРЭ. [7-8]

Нарушение сохранности информации в ячейках памяти и/или нарушение работы управляющих узлов регистрируется как ФС, который может привести к потере информации, хранящейся в ячейках памяти, что в последствии может привести к сбоям систем управления космического назначения $[2,9,10]$ и разработчику аппаратуры критически необходимо знать характер каждого из ФС для разработки системы парирования наблюдаемых эффектов в аппаратуре для увеличения безопасности хранящейся информации.

Микросхемы flash-памяти используются в системах космического назначения для хранения кодов программ и данных в аппаратуре. Преимуществами flash-памяти является энергонезависимость, возможность перепрограммирования и большая ёмкость памяти.

В связи с постоянным развитием микросхем, добавляется более гибкое конфигурирование, добавляются расширенные настройки, а также совершенствуются существующие интерфейсы и появляются новые, необходимо адаптировать имеющуюся методику контроля ФС для определения параметров чувствительности микросхем к воздействию ОЯЧ по ОРЭ.

\section{1. Контроль функциональных сбоев при исследованиях микросхем nor flash-памяти}

Основной причиной нарушений сохранности информации в микросхемах flashпамяти является потеря заряда с элемента хранения транзистора ячейки. В результате потери заряда происходит сдвиг порогового напряжения транзистора ячейки, и управляющая схема воспринимает ячейку как стёртую [9]. 
Роман И. Гвоздев, Иван И. Швецов-Шиловский, Сергей Б. Шмаков.

АДАПТИРОВАННАЯ МЕТОДИКА КОНТРОЛЯ ФУНКЦИОНАЛЬНЫХ СБОЕВ

В NOR FLASН-ПАМЯТИ ПРИ ИСПЫТАНИЯХ НА СТОЙКОСТЬ

К ВОЗДЕЙСТВИЮ ТЯЖЁЛЫХ ЗАРЯЖЕННЫХ ЧАСТИЦ

ФС при воздействии ОЯЧ определяются по реакции микросхемы в процессе выполнения командных циклов. Командный цикл - это цикл, на протяжении которого выполняется команда, отправленная в микросхему. При контроле командных циклов используются управляющие регистры. С помощью опроса регистров во время выполнения командного цикла, можно отследить его время выполнения, что является параметром функционирования в технической документации $[2,9]$.

В процессе опроса регистров, возможно нарушение сохранности данных управляющих регистров (энергозависимых и энергонезависимых), что регистрируется как ФС, который приводит к нарушению установленных режимов работы микросхемы (нарушение установленного режима ввода/вывода данных, блокировка секторов памяти для операций стирания/записи). Также, во время функционирования микросхемы, ФС проявляется как спонтанный запуск командных циклов операций записи/считывания/стирания, сопровождающиеся резким увеличением/уменьшением тока потребления микросхемы, который восстанавливался до исходного значения самопроизвольно или после отправки команды программного сброса микросхемы.

Во время считывания проявлением одиночного сбоя (ОС) является ошибка в полученном коде. Для восстановления функционирования необходимо перезаписать ячейки памяти, в которых произошёл сбой, или повторно считать информационный код. При регистрации сбоя считывания все последующие циклы могут быть с ошибками, поэтому требуется провести программный сброс или выключение питания микросхемы [9].

Сбой, во время выполнения командного цикла стирания/записи предлагается определять с помощью управляющих регистров: бит отвечающий за выполнение операции стирания/записи не сбрасывается в состояние «Готов» по прошествии максимального времени выполнения командного цикла стирания/записи, указанного в технической документации. Также ток в цепи питания резко падает. Чтобы восстановить функционирование проводится программный сброс или выключение питания, или перезапуск операции стирания [9, 11, 12].

Запуск спонтанного стирания памяти проявляется как потеря информации и ток потребления возрастает до значения равному при выполнении операции стирания. Повторная запись информационного кода устранит сбой [9].

\section{2. Методика и аппаратно-программные средства контроля функциональных сбоев}

Испытания электронной компонентной базы (ЭКБ) на стойкость к ОРЭ при воздействии ИИ проводятся на ускорителях ионов, протонов и лазерных установках со сфокусированным излучением. ОРЭ в изделиях ЭКБ, вызываемые воздействием ИИ, считают адекватными по признаку идентичности ионизационных реакций параметрических и/или ФС и отказов, определяемых по внешним выводам изделий. Во время проведения испытаний, при фиксированном значении линейных потерь энергии ионов регистрируются ОРЭ.

На рис. 1 представлена адаптированная методика проведения исследований стойкости flash-памяти к воздействию ОЯЧ по эффектам ФС $[9,11,13]$.

Контроль тиристорного эффекта (ТЭ) при испытательном воздействии проводится в активном режиме, с контролем тока потребления и контролем работоспособности. Пороговый уровень ТЭ устанавливается в зависимости от среднего уровня тока потребления, с учетом изменений тока при изменении режимов работы. При регистрации катастрофического отказа (КО) в каком-либо из режимов облучение микросхемы прекращалось. 
Роман И. Гвоздев, Иван И. Швецов-Шиловский, Сергей Б. Шмаков.

АДАПТИРОВАННАЯ МЕТОДИКА КОНТРОЛЯ ФУНКЦИОНАЛЬНЫХ СБОЕВ

В NOR FLASН-ПАМЯТИ ПРИ ИСПЫТАНИЯХ НА СТОЙКОСТЬ К ВОЗДЕЙСТВИЮ ТЯЖЁЛЫХ ЗАРЯЖЕННЫХ ЧАСТИЦ

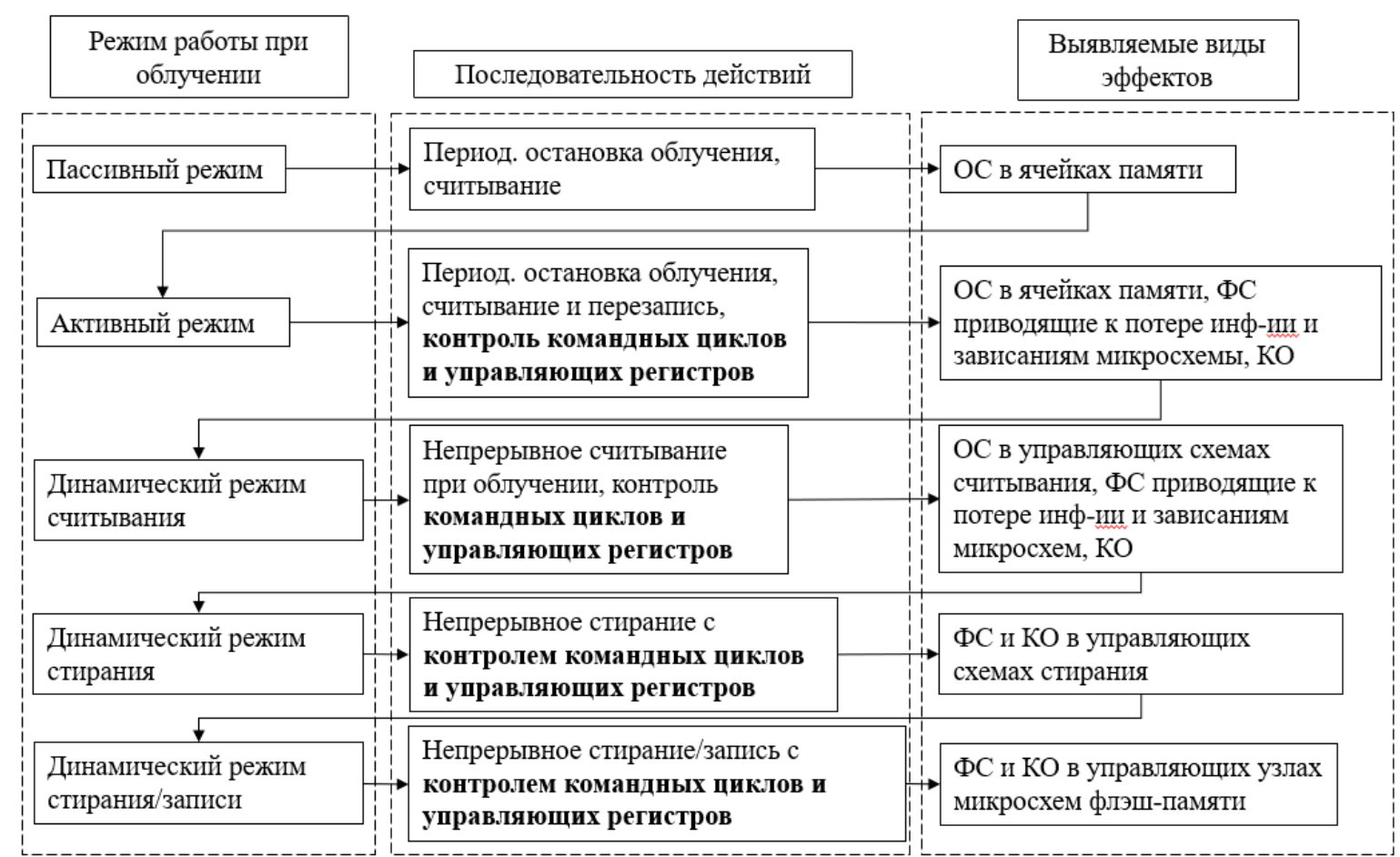

Рис. 1. Методика проведения исследований стойкости флэи-памяти к воздействию ОЯЧ по эффектам функииональных сбоев

(Fig. 1. Methods for conducting research on the resistance of flash memory to the effects of SNP on the effects of functional failures)

В методику добавлены дополнительные действия во время исследований, а именно: контроль командных циклов и управляющих регистров. Дополненная последовательность действий во время исследований позволит контролировать выполнения операций и режимов работы, в которых находится микросхема.

\section{3. Аппаратно-программная реализация средств контроля функциональных сбоев}

Для реализации контроля командных циклов и управляющих регистров адаптирован стандартный аппаратно-программный комплекс, разработанный на основе оборудования фирмы National Instruments, структурная схема которого представлена на рис. 2. Особенностью является обмен данными с использованием расширенного интерфейса SPI по четырём линиям ввода/вывода (Quad SPI protocol). Комплекс подходит для микросхем NOR flash-памяти с интерфейсом SPI, у которых предусмотрена возможность обмена данными по четырём линиям ввода/вывода.

На рис. 2 введены следующие обозначения: G1 - источник питания (PXI-4110); G2 - вспомогательный источник напряжения (PXI-6229); A1, А2 - измерители тока PXI-4171 и PXI-4071; V2 - вольтметр; К1 размыкается только при контроле тока потребления; К2 замыкается только при контроле выходного напряжения; $\mathrm{R} 1=510$ Ом; $\mathrm{C} 1=0,1$ мкФ; $\mathrm{Q} 0: \mathrm{Q}(\mathrm{N}-1)$ - сигнальные линии ввода/вывода; N - количество линий ввода/вывода (всего можно использовать от 1 до 4 линий); $\mathrm{S \#} \mathrm{-} \mathrm{выбор} \mathrm{устройства;} \mathrm{C} \mathrm{-} \mathrm{тактовый} \mathrm{сигнал.}$ 


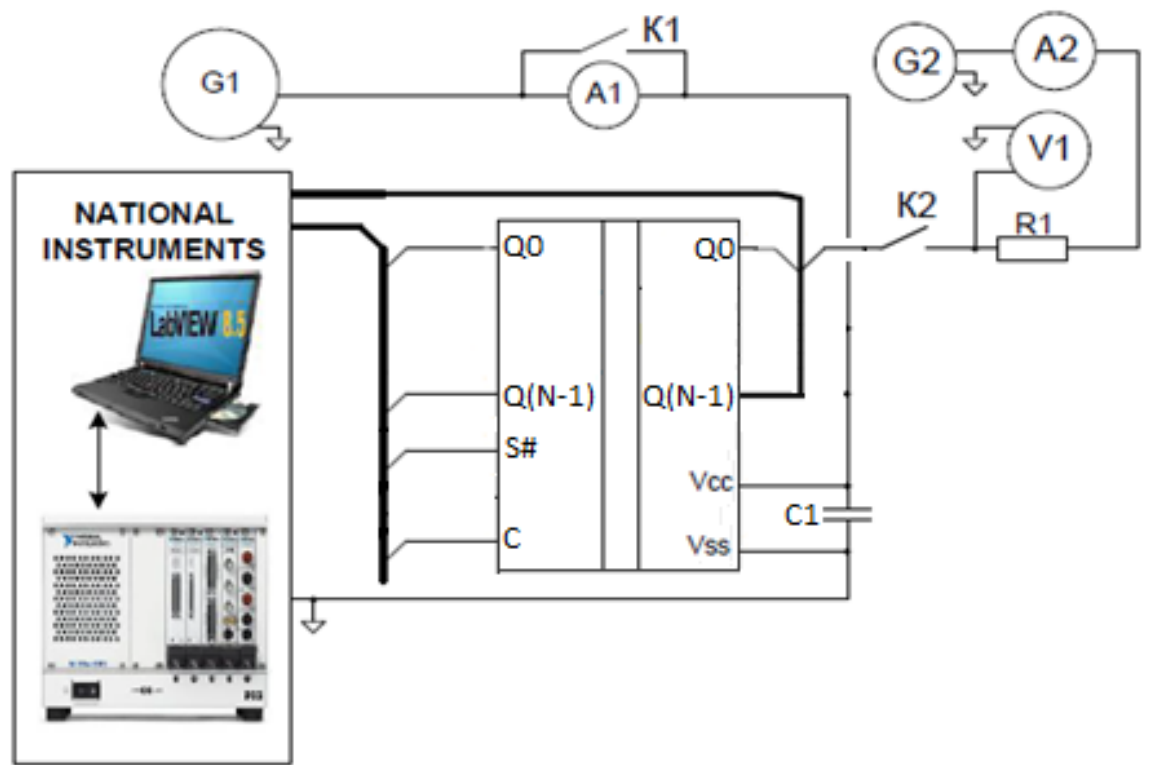

Рис. 2. Структурная схема аппаратно-программного комплекса (Fig. 2. Block diagram of a hardware-software complex)

Контроль выходных напряжений осуществляется вольтметром V1 на выводе Q0. Режимный ток выхода устанавливается регулировкой напряжения вспомогательного источника G2 на резисторе R1 и контролируется измерителем тока A2.

Ключ К1 предназначен для переключения линии цепи питания, если требуется измерить потребляемый микросхемой ток. Ключ К2 предназначен для переключения линии ввода/вывода Q0 на вольтметр V1 при измерении выходных напряжений.

В аппаратно-программном комплексе используются следующие аппаратные средства:

- PXI-1033 - 5-слотовое шасси. Предназначено для управления модулями через персональный компьютер.

- Модуль PXI-4110 - 3-канальный программируемый источник питания постоянного тока. Имеет 3 канала питания с ограничением до 1 А на каждый канал. Используется для питания микросхемы, а также питания цифрового модуля РXI-6581.

- Модуль РХІ-4071 - цифровой мультиметр. Используется для контролирования тока в цепи питания.

- Модуль PXI-7951R - используется вместе с адаптерным модулем PXI-6581.

- Модуль PXI-6581 - 54-канальный цифровой адаптер ввода/вывода. Совместно с PXI-7951R создают настраиваемый прибор цифрового ввода-вывода, который можно запрограммировать с помощью ПО LabVIEW FPGA для взаимодействия с микросхемой. Используется для взаимодействия с микросхемами в режиме реального времени с частотой до 100 МГц.

- Модуль РХІ-6229 - многофункциональный модуль ввода/вывода, имеющий 32 аналоговых входа, 4 аналоговых выхода и 48 цифровых вводов-выводов. Используется как преобразователь напряжения для задания нагрузочного тока и контроля выходных напряжений. 
Контроль выходных напряжений $\mathrm{V}_{\mathrm{H}}$ и $\mathrm{V}_{\mathrm{L}}$ осуществлялся с помощью PXI-6229 на выводе Q0 в режиме статической выборки логического 0 или логической 1 до и после облучения.

Контроль тока потребления во время облучения проводился с помощью измерителя тока, совмещённого с источником питания РXI-4110.

Контроль тока потребления $\left(\mathrm{I}_{1}, \mathrm{I}_{2}\right)$ до и после облучения осуществлялся с измерителем тока потребления РXI-4071.

Взаимодействие с микросхемами обеспечивается с помощью персонального компьютера с разработанным программным обеспечением в среде LabVIEW.

На рис. 3 приведена блок-схема адаптированной управляющей программы, где представлены функции и подфункции, из которых состоят части «Host» и «Target». Программа является конечным автоматом вместе с дополнительными функциями, которые выполняются параллельно (выделено жирно).

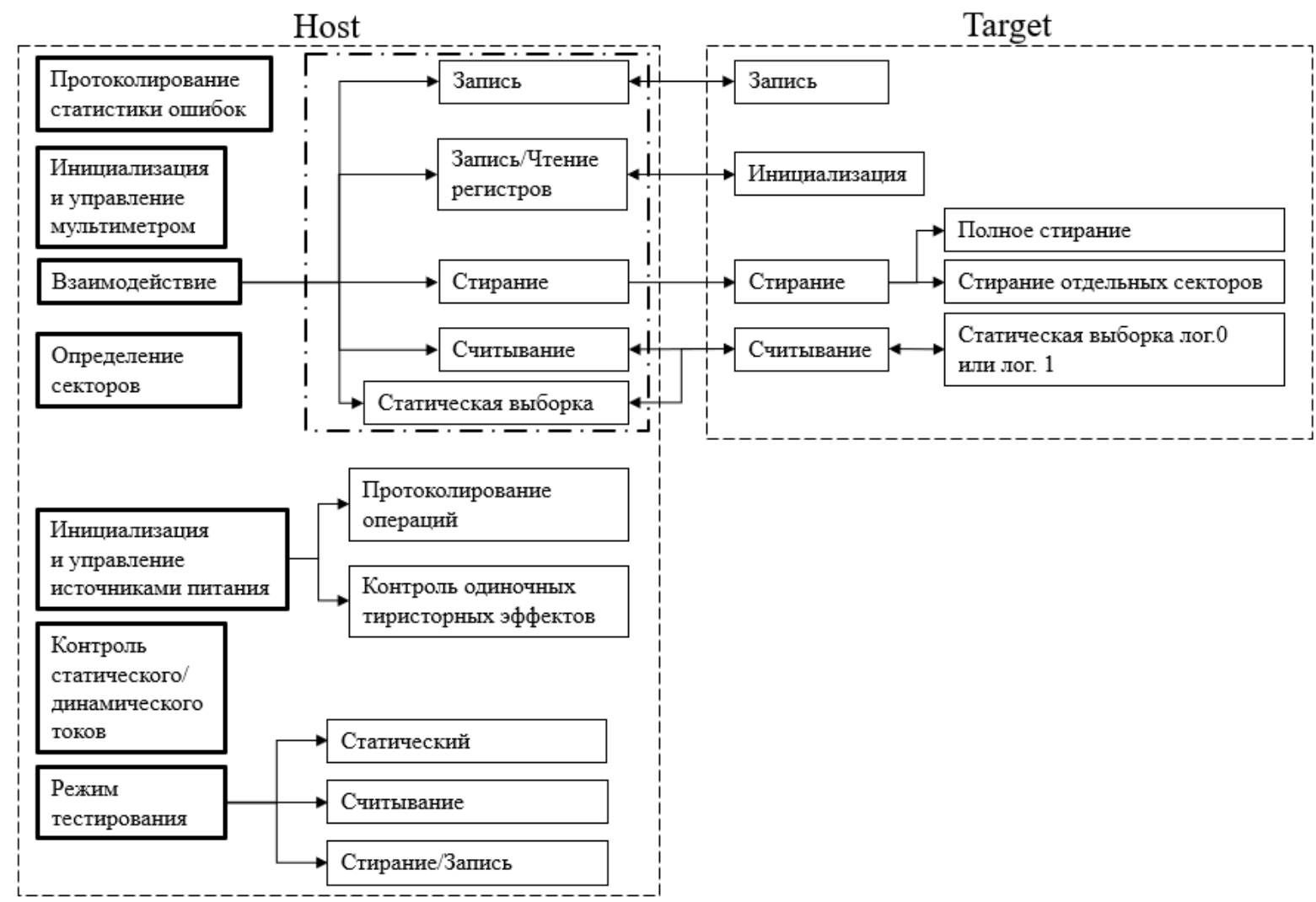

Рис. 3. Блок-схема управляющей программы

(Fig. 3. The block diagram of the control program)

Программа представляет собой многофункциональный интерфейс управления для взаимодействия с микросхемой, поддерживающий следующие основные операции:

- Запись всего объёма памяти эталонным кодом

- Считывание всего объёма памяти

- Стирание всего объёма памяти

- Сравнение считанных данных с эталонным кодом

- Контроль динамического и статического токов потребления

- Контроль выходных логических уровней

- Контроль одиночных тиристорных эффектов 
Также реализованы дополнительные функции:

- Стирание отдельных секторов

- Считывание отдельных секторов

- Запись отдельных секторов эталонным кодом

- Запись/считывание управляющих регистров

Объектом исследований являются микросхемы flash-памяти NOR-типа информационной ёмкостью 256 Мбит с интерфейсом SPI. Микросхема изготовлена по технологии 65 нм. Диапазон напряжений питания от 2,7 В до 3,6 В.

В микросхеме реализована постраничная запись данных. Каждая страница может быть индивидуально запрограммирована. Биты программируются из единицы в ноль. Имеется возможность стереть подсектор, сектор или всю память. Всего в памяти 33554432 байта; 512 секторов (64 Кбайт каждый); 8192 подсектора (4 Кбайт каждый); 131072 страницы (256 байт каждая) и 64 однократно программируемых байта, расположенных отдельно от остальной памяти.

Поддерживается 3-х байтный и 4-х байтный адресный режим.

Устройство имеет энергозависимые и энергонезависимые регистры, к которым пользователь может получить доступ, а именно:

- Регистр статуса - используется для контроля командного цикла записи и запрета записи/стирания.

- Энергозависимые и энергонезависимые регистры конфигурации - используются для включения/выключения протоколов интерфейса SPI.

- Адресный регистр - используется для переключения между верхним и нижним сегментом памяти при использовании 3-х байтного адреса.

- Регистр флагов - используется для контроля командного цикла стирания.

\section{4. Порядок проведения и результаты эксперимента}

Эксперимент проводился на базе циклотрона У-400 (ЛЯР ОИЯИ, г. Дубна Московской области). Последовательность режимов во время эксперимента при испытательном воздействии:

- Статический режим с периодическим контролем работоспособности.

- Режим считывания с контролем сохранности данных конфигурирующих регистров и командных циклов.

- Режим стирания/записи с контролем сохранности данных конфигурирующих регистров и командных циклов.

Контроль функционирования во время эксперимента заключался в проверке сохранности информации, а также возможности её перезаписи. Тестирование микросхемы проводилось в следующем порядке:

- Инициализация микросхемы эталонным кодом («инкрементный», «декрементный»).

- Считывание записанной информации, сравнение с эталонным значением.

Экспериментальные результаты показали, что необходимо разделить функциональные сбои на несколько типов, так как различалась реакция микросхемы при взаимодействии. Зарегистрированные функциональные сбои представлены в табл. 1. 
Роман И. Гвоздев, Иван И. Швецов-Шиловский, Сергей Б. Шмаков.

АДАПТИРОВАННАЯ МЕТОДИКА КОНТРОЛЯ ФУНКЦИОНАЛЬНЫХ СБОЕВ

В NOR FLASН-ПАМЯТИ ПРИ ИСПЫТАНИЯХ НА СТОЙКОСТЬ К ВОЗДЕЙСТВИЮ ТЯЖЁЛЫХ ЗАРЯЖЕННЫХ ЧАСТИЦ

Таблииа 1. Тип и критерии зарегистрированных ФС во время эксперимента

\begin{tabular}{|c|c|c|}
\hline $\begin{array}{l}\text { Тип } \\
\text { ФС }\end{array}$ & $\begin{array}{c}\text { Реакция микросхемы на команды } \\
\text { считывания или стирания }\end{array}$ & $\begin{array}{c}\text { Предпринятые действия } \\
\text { для восстановления функционирования }\end{array}$ \\
\hline$\Phi C 1$ & Отсутствие реакции на команды & $\begin{array}{l}\text { Перезапись энергозависимых } \\
\text { управляющих регистров }\end{array}$ \\
\hline ФС2 & $\begin{array}{l}\text { Нарушение сохранности } \\
\text { информации в блоке памяти } \\
\text { размеров } 524288 \text { бит }\end{array}$ & $\begin{array}{l}\text { Стирание блока памяти и повторная } \\
\text { запись эталонным кодом }\end{array}$ \\
\hline ФС3 & $\begin{array}{l}\text { Отсутствие реакции микросхемы } \\
\text { на команду Bulk Erase (полное } \\
\text { стирание накопителя) }\end{array}$ & $\begin{array}{l}\text { Опрос регистра статуса. В случае } \\
\text { активации запрета стирания/записи } \\
\text { перезаписать регистр }\end{array}$ \\
\hline$\Phi C 4$ & $\begin{array}{l}\text { Увеличение тока потребления } \\
\text { 150мА с самопроизвольным } \\
\text { восстановлением }\end{array}$ & Запуск программного сброса \\
\hline ФС5 & $\begin{array}{l}\text { Увеличение тока потребления до } \\
\text { уровня 5-50мА с } \\
\text { восстановлением после подачи } \\
\text { команд или сброса питания }\end{array}$ & Запуск программного сброса \\
\hline
\end{tabular}

\section{Заключение}

Апробирована адаптированная методика контроля ФС для определения параметров чувствительности микросхем к воздействию ОЯЧ по ОРЭ.

В процессе подготовки к эксперименту адаптирован стандартный аппаратнопрограммный комплекс, разработанный на основе оборудования фирмы National Instruments.

Рассмотрены возникающие ФС по контролю командных циклов и управляющих регистров. Экспериментальные результаты апробации адаптированной методики показали необходимость разделения ФС на 5 типов в зависимости от реакции микросхемы на команды.

В процессе эксперимента обнаружен сбой регистра статуса (ФС3). Разряды отвечающие за запрет операции записи/стирания сбивались, что приводило к блокировке команды полного стирания накопителя (Bulk Erase), микросхема не реагировала на команду. Без контроля сохранности данных в регистре, такая реакция считалась бы катастрофическим отказом микросхемы, так как одна из операций стала недоступна.

Также обнаружен сбой конфигурирующего регистра (ФС1). Разряды, отвечающие за режимы работы интерфейса SPI, сбивались, что приводило к неправильной работе управляющей программы, так как в микросхеме изменился режим работы интерфейса. Контролирование управляющего регистра способствовало улучшению функционального контроля микросхемы при облучении.

\section{СПИСОК ЛИТЕРАТУРЫ:}

1. Чумаков А.И. «Действие космической радиации на интегральные схемы». М.: Радио и связь. 2004. 320 c. URL: https://www.elibrary.ru/item.asp?id=19635287 (дата обращения: 29.07.2020).

2. Чумаков, А.И. Радиационная стойкость изделий ЭКБ [Текст] / А.И. Чумаков // Общая характеристика ионизирующих излучений: сб. статей. - М.: НИЯУ МИФИ, 2015. - 512 с.

3. Беляков В.В., Чумаков А.И., Никифоров А.Ю., Першенков В.С., Скоробогатов П.К., Согоян А.В. Расчётно-экспериментальные методы прогнозирования эффектов одиночных сбоев в элементах современной микроэлектроники // Микроэлектроника, 2003. Т. 32, № 2. С. 134-151. URL: https://www.elibrary.ru/item.asp?id=17262938 (дата обращения: 29.07.2020). DOI: https://doi.org/10.1023/A:1022656102956. 
Роман И. Гвоздев, Иван И. Швецов-Шиловский, Сергей Б. Шмаков.

АДАПТИРОВАННАЯ МЕТОДИКА КОНТРОЛЯ ФУНКЦИОНАЛЬНЫХ СБОЕВ

В NOR FLASН-ПАМЯТИ ПРИ ИСПЫТАНИЯХ НА СТОЙКОСТЬ

К ВОЗДЕЙСТВИЮ ТЯЖЁЛЫХ ЗАРЯЖЕННЫХ ЧАСТИЦ

4. Sogoyan A.V., Chumakov A.I. and Smolin A.A., "Single Event Rate Prediction Method for Advanced CMOS Technologies," 2018 International Conference on Radiation Effects of Electronic Devices (ICREED), Beijing, China, 2018. P. 1-5. DOI: https://doi.org/10.1109/ICREED.2018.8905071.

5. A.B. Boruzdina, A.V. Yanenko, A.V. Ulanova, A.I. Chumakov, D.V. Bobrovskiy and V.M. Uzhegov, "Microdose effects in SRAM cells under heavy ion irradiation," 2017 17th European Conference on Radiation and Its Effects on Components and Systems (RADECS), Geneva, Switzerland, 2017. P. 1-3, DOI: https://doi.org/10.1109/RADECS.2017.8696109.

6. A.A. Pechenkin, A.B. Boruzdina, A.V. Yanenko, D.E. Protasov, I.I. Shvetsov-Shilovskiy and A.A. Sangalov, "SEL and cell failures in MRAM under ion and focused laser irradiation," 2017 17th European Conference on Radiation and Its Effects on Components and Systems (RADECS), Geneva, Switzerland, 2017. P. 1-6. DOI: https://doi.org/10.1109/RADECS.2017.8696211.

7. Чумаков, Александр И. и др. Требования и нормы испытаний по радиационной стойкости интегральных схем к эффектам воздействия тяжёлых заряженных частиц. Безопасность информационных технологий, [S.1.], T. 27, №. $1 . \quad$ С. $83-97$, фев. 2020. URL: https://bit.mephi.ru/index.php/bit/article/view/1254 (дата 18.08.2020). DOI: http://dx.doi.org/10.26583/bit.2020.1.07.

8. Чумаков А.И. и др. Оценка показателей стойкости интегральных схем при воздействии тяжелых заряженных частиц с использованием различных моделей. Безопасность информационных технологий, [S.1.]. T. 24, № 1. C. 73-84, 2017. ISSN 2074-7136. URL: https://bit.mephi.ru/index.php/bit/article/view/58 (дата обращения: 18.08.2020). DOI: http://dx.doi.org/10.26583/bit.2017.1.09.

9. Петров, А.Г. Функциональные отказы в микросхемах флэш-памяти от воздействия ионизирующих излучений космического пространства [Текст]: автореф. дис. на соиск. учен. степ. канд. техн. наук (05.13.05) / Петров Андрей Григорьевич; НИЯУ МИФИ - Москва, 2014 - 22 с. URL: https://www.elibrary.ru/item.asp?id=30416686 (дата обращения: 29.07.2020).

10. R. Koga, S. H. Penzin, K. B. Crawford and W. R. Crain, "Single event functional interrupt (SEFI) sensitivity in microcircuits," RADECS 97. Fourth European Conference on Radiation and its Effects on Components and Systems (Cat. No.97TH8294), Cannes, France, 1997. P. 311-318. DOI: https://doi.org/10.1109/RADECS.1997.698915.

11. Петров А.Г., Яненко А.В., Васильев А.Л., Чумаков А.И. «Поведение тока потребления микросхем флэш-памяти при возникновении эффектов сбоев типа SEFI» // Радиационная стойкость электронных систем - «Стойкость-2013», вып. 16, Москва, 2013. С. $163-164$. URL: http:/www.spels.ru/index.php?option=com_content\&view=article\&id=179\&Itemid=73 (дата обращения: 18.08.2020) (in Russian).

12. Петров А.Г. «Регистрация функциональных сбоев, приводящих к стиранию информации во флэш $3 У$ при воздействии одиночных ядерных частиц». URL: https:/www.elibrary.ru/item.asp?id=25658477 (дата обращения: 18.08.2020).

13. Петров А.Г., Уланова А.В., Чумаков А.И., Васильев А.Л. Исследования потери информации в микросхемах флэш-памяти в активном и пассивном режимах при ионизирующем воздействии // Радиационная стойкость электронных систем - «Стойкость-2014», вып.17, Москва, 2014. С. $175-176$. URL: http:/www.spels.ru/index.php?option=com_content\&view=article\&id=179\&Itemid=73 (дата обращения: 18.08.2020).

\section{REFERENCES:}

[1] Chumakov A.I. Dejstvie kosmicheskoj radiacii na integral'nye shemy [Tekst]. Moskva.: Radio i svjaz', 2004. 320 s. URL: https://www.elibrary.ru/item.asp?id=19635287 (in Russian).

[2] Chumakov A.I. Radiation resistance of electronic component base products. General characteristics of ionizing radiation: collection of articles., Moscow: National research nuclear University MEPhI, 2015. - 512 p. (in Russian).

[3] Beljakov V.V., Chumakov A.I., Nikiforov A.Ju., Pershenkov V.S., Skorobogatov P.K., Sogojan A.V. Raschjotno-jeksperimental'nye metody prognozirovanija jeffektov odinochnyh sboev $\mathrm{V}$ jelementah sovremennoj mikrojelektroniki. Mikrojelektronika, 2003. T. 32, № 2. S. $134-151$. URL: $\quad$ https://www.elibrary.ru/item.asp?id=17262938 (дата обращения: 29.07 .2020 ). DOI: https://doi.org/10.1023/A:1022656102956. (in Russian).

[4] Sogoyan A.V., Chumakov A.I. and Smolin A.A., "Single Event Rate Prediction Method for Advanced CMOS Technologies," 2018 International Conference on Radiation Effects of Electronic Devices (ICREED), Beijing, China, 2018. P. 1-5. DOI: https://doi.org/10.1109/ICREED.2018.8905071.

[5] A.B. Boruzdina, A.V. Yanenko, A.V. Ulanova, A.I. Chumakov, D.V. Bobrovskiy and V.M. Uzhegov, "Microdose effects in SRAM cells under heavy ion irradiation," 2017 17th European Conference on Radiation 
Роман И. Гвоздев, Иван И. Швецов-Шиловский, Сергей Б. Шмаков.

АДАПТИРОВАННАЯ МЕТОДИКА КОНТРОЛЯ ФУНКЦИОНАЛЬНЫХ СБОЕВ

В NOR FLASН-ПАМЯТИ ПРИ ИСПЫТАНИЯХ НА СТОЙКОСТЬ К ВОЗДЕЙСТВИЮ ТЯЖЁЛЫХ ЗАРЯЖЕННЫХ ЧАСТИЦ

and Its Effects on Components and Systems (RADECS), Geneva, Switzerland, 2017. P. 1-3, DOI: https://doi.org/10.1109/RADECS.2017.8696109.

[6] A.A. Pechenkin, A.B. Boruzdina, A.V. Yanenko, D.E. Protasov, I.I. Shvetsov-Shilovskiy and A.A. Sangalov, "SEL and cell failures in MRAM under ion and focused laser irradiation," 2017 17th European Conference on Radiation and Its Effects on Components and Systems (RADECS), Geneva, Switzerland, 2017. P. 1-6. DOI: https://doi.org/10.1109/RADECS.2017.8696211.

[7] Chumakov Aleksandr I. et al. Hardness assurance levels and requirements for single event effects testing of integrated circuits. IT Security (Russia), [S.1.]. V. 27, no. 1. P. 83-97, feb. 2020. URL: $\quad$ https://bit.mephi.ru/index.php/bit/article/view/1254 18.08.2020). DOI: http://dx.doi.org/10.26583/bit.2020.1.07 (in Russian)

[8] Chumakov Aleksandr I. et al. Single Event Effects Rate Calculation with Different Models. IT Security

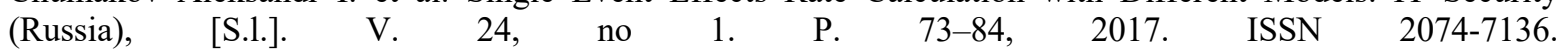
URL: $\quad$ https://bit.mephi.ru/index.php/bit/article/view/58 18.08.2020). DOI: http://dx.doi.org/10.26583/bit.2017.1.09 (in Russian).

[9] Petrov A.G. Funkcional'nye otkazy v mikroshemah fljesh-pamjati ot vozdejstvija ionizirujushhih izluchenij kosmicheskogo prostranstva [Tekst]: avtoref. dis. na soisk. uchen. step. kand. tehn. nauk (05.13.05). Petrov Andrej Grigor'evich; NIJaU MIFI - Moskva, $2014-22$ s. URL: https://www.elibrary.ru/item.asp?id=30416686 (accessed: 29.07.2020) (in Russian).

[10] R. Koga, S.H. Penzin, K.B. Crawford and W. R. Crain, "Single event functional interrupt (SEFI) sensitivity in microcircuits," RADECS 97. Fourth European Conference on Radiation and its Effects on Components and Systems (Cat. No.97TH8294), Cannes, France, $1997 . \quad$ P. 311-318. DOI: https://doi.org/10.1109/RADECS.1997.698915.

[11] Petrov A.G., Yanenko A.V., Vasil'ev A.L., Chumakov A.I. Povedenie toka potrebleniya mikroskhem fleshpamyati pri vozniknovenii effektov sboev tipa SEFI. Radiacionnaya stojkost' elektronnyh sistem - «Stojkost'-

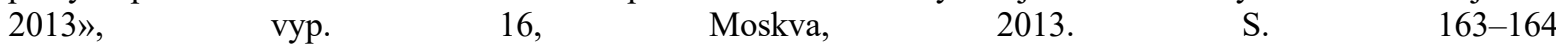
URL: http://www.spels.ru/index.php?option=com_content\&view=article\&id=179\&Itemid=73 (accessed: 18.08.2020) (in Russian).

[12] Petrov A.G. «Registracija funkcional'nyh sboev, privodjashhih k stiraniju informacii vo fljesh ZU pri vozdejstvii odinochnyh jadernyh chastic». URL: https:/www.elibrary.ru/item.asp?id=25658477 (accessed: 18.08.2020) (in Russian).

[13] Petrov A.G., Ulanova A.V., Chumakov A.I., Vasil'ev A.L. Issledovanija poteri informacii v mikroshemah fljesh-pamjati v aktivnom i passivnom rezhimah pri ionizirujushhem vozdejstvii // Radiacionnaja stojkost' jelektronnyh sistem - «Stojkost'-2014», vyp. 17, Moskva, 2014. S. $175-176$. URL: http://www.spels.ru/index.php?option=com_content\&view=article\&id=179\&Itemid=73 (accessed: 18.08.2020) (in Russian).

Поступила в редакцию - 26 июля 2020 г. Окончательный вариант - 20 августа 2020 г. Received - July 26, 2020. The final version - August 20, 2020. 